\title{
Changes of hepatic proteome in bile duct ligated rats with hepatic fibrosis following treatment with Yin-Chen-Hao-Tang
}

\author{
TZUNG-YAN LEE ${ }^{1}$, HEN-HONG CHANG ${ }^{1,3}$, JONG-JEN KUO $^{3}$ and JIANN-JONG SHEN ${ }^{2,3}$ \\ ${ }^{1}$ Graduate Institute of Traditional Chinese Medicine, ${ }^{2}$ School of Chinese Medicine, Chang Gung University; \\ ${ }^{3}$ Center for Traditional Chinese Medicine, Chang Gung Memorial Hospital, Taoyuan, Taiwan, R.O.C.
}

Received November 27, 2008; Accepted February 9, 2009

DOI: 10.3892/ijmm_00000154

\begin{abstract}
Yin-Chen-Hao-Tang (YCHT) is recognized as a hepatoprotective agent for various types of liver diseases. Proteomics approaches were used to study hepatic and serum protein expression changes in bile duct ligated (BDL) rats following YCHT treatment for 27 days. Two-dimensional gel electrophoresis was used to analyze proteome changes. Of the proteins that exhibited changes, 17 were identified by means of matrix-assisted laser desorption/ionization-time-offlight (MALDI-TOF) mass spectrometry. The major effect of YCHT was evident in cytoskeleton related protein, plectin-1. In addition, proteins involved in metabolism of lipids were also shown to be affected, including low-density lipoprotein receptor-related protein 2 precursor (glycoprotein 330) and apolipoprotein A-I precursor (ApoA-I). Significant upregulation of keratin 8 and 19 was found in liver tissue of BDL rats. Supplementation with YCHT also triggered alterations in the above proteins. Interestingly, YCHT treatment caused a statistically significant down-regulation in the secretion of monocyte chemoattractant protein-1 (MCP-1) and tissue inhibitor of metalloproteinase-1 (TIMP-1) in BDL rats with fibrosis. Our results suggested that YCHT may be useful for treatment of liver fibrosis because of its possible antiapoptotic properties, and the therapeutic effects of YCHT on liver diseases might be associated with its lipid biosynthesis regulation.
\end{abstract}

\section{Introduction}

Liver fibrosis results from chronic damage to the liver in conjunction with accumulation of extracellular matrix (ECM) proteins, which is a characteristic of most types of chronic liver diseases (1). Decreased activity of ECM-removing, matrix metalloproteinases (MMPs) is mainly due to overexpression of their specific inhibitors (2). Before fibrosis and cirrhosis

Correspondence to: Dr Tzung-Yan Lee, Graduate Institute of Traditional Chinese Medicine, Chang Gung University, No. 259, Wen-Hwa 1st Road, Kwei-Shan Tao-Yuan 333, Taiwan, R.O.C. E-mail: joyamen@mail.cgu.edu.tw

Key words: hepatic fibrosis, proteomics, Yin-Chen-Hao-Tang can be easily observed at the histological level, many changes in the secretion profile of the hepatic fibroblasts are thought to occur. Therefore, proteomic technologies can be used to identify protein markers for the early detection of fibrosis associated events.

Tissue proteome analysis has been successfully applied to investigate the molecular effects of drugs and to obtain information regarding their mode of action and therapeutic mechanism $(3,4)$. In addition, proteomic technologies have been applied to study changes in the levels of liver proteins of mice treated with acetaminophen (5) and of rats treated with carbon tetrachloride (6). Yin-Chen-Hao-Tang (Inchinko-to or TJ-135 in Japan) consists of the following three medicinal herbs: Artemisia capillaries Thunb, Gardenia jasminoides Ellis, and Rheum officinale Baill. This decoction has long been used in Taiwan and China as an anti-inflammatory, antipyretic, and choleretic agent for liver disorders and jaundice. In the literature, a variety of biological effects of YCHT have been described. This decoction has been reported to show a potent effect on hepatic fibrosis (7-10) and hepatic apoptosis in vitro (11) and in vivo $(12,13)$.

The aim of our study was a systemic comparison of all hepatic protein changes during BDL stress and further analyzed the proteome changes of BDL rats after YCHT treated. In this study, we also demonstrated that YCHT may modulate hepatic TIMP-1 and MMP-2 expression through decreased ECM accumulation. Secondly, we examined the Chinese herbal prescription and found possible complex pattern of alterations in BDL rat liver proteins, some of which were related to lipid metabolism, but many represented effects on the filamentous network pathways and endpoints.

\section{Materials and methods}

Preparation of YCHT. YCHT was prepared as described in our previous studies (13). Briefly, YCHT extract powder consists of crude ingredients extracted from the following three medicinal herbs mixed in the following ratio: Artemisia capillaries Thunb, Gardenia jasminoides Ellis, and Rheum officinale Baill, 4:3:1 (by weight). The three main index components of the YCHT decoction were identified by high performance liquid chromatography (HPLC) by comparing the retention times and UV spectra as $0.95 \%$ (w/w) geniposide, $0.14 \%$ capillarisin and $0.11 \%$ emodin. The analytical column was a Cosmosil C18 column (250x4.6 mm internal diameter). 
The column temperature was maintained at $40^{\circ} \mathrm{C}$. The HPLC mobile phase was a 65:35 mixture of water and acetonitrile containing phosphoric acid $(0.03 \% \mathrm{v} / \mathrm{v})$, at a flow rate of $1.0 \mathrm{ml} / \mathrm{min} ; 20 \mathrm{ml}$ of solution was injected into the HPLC system for analysis. Peaks correlating with geniposide, capillarisin and emodin represented G. jasminoides Ellis, A. capillaris Thunb, and R. officinale Baill, respectively.

Biliary obstruction and animal treatments. Adult male SpragueDawley rats weighing between 220 and $250 \mathrm{~g}$ were used in all experiments. Hepatic damage with fibrosis was produced by common BDL, as we previously described (10). All rats were caged at $24^{\circ} \mathrm{C}$ with a $12: 12 \mathrm{~h}$ light-dark cycle, and were allowed free access to food and water. Animal studies were approved by the Animal Experiment Committee of the Chang Gung University, and were conducted humanely. After surgery, YCHT was dissolved in distilled water and administered daily ( $250 \mathrm{mg} / \mathrm{kg}$ p.o.) over the experimental period. Sham operated rats received equal volumes of the distilled water.

Histopathology assay and cytokine array assay. The liver tissue was fixed in $10 \%$ formalin and then embedded in paraffin, cut into $5 \mu \mathrm{m}$ thick sections, stained with Masson's trichrome, and examined under light microscopy by an experienced pathologist. Serum was obtained for cytokine array. Cytokine array assay was carried out with a RayBio ${ }^{\mathrm{TM}}$ rat cytokine array kit (RayBiotech, Inc., Norcross GA) according to the manufacturer's protocol. Each cytokine array membrane was scanned, and the intensities of signals were quantitated by densitometry (Bio-Rad, Hercules, CA). The supernatants were collected by centrifugation at $3000 \mathrm{rpm}$ for $15 \mathrm{~min}$. Albumin or IgG was removed from the supernatants using the aurum serum protein mini kit (Bio-Rad), and the supernatants were concentrated by centrifugation at $5000 \mathrm{x} \mathrm{g}$ using an Ultrafree-MC PLCC centrifugal filter unit $5 \mathrm{kDa}$ membrane (Millipore, Billerica, MA) to $25 \%$ of the original volume.

Reverse transcription-polymerase chain reaction ( $R T-P C R)$ amplification of TIMP-1 and MMP-2. Liver (100 mg) was snap-frozen in liquid nitrogen and homogenized in $1 \mathrm{ml}$ of TRIzol solution (BRL, Gaithersburg, MD). Total cellular RNA was extracted according to the manufacturer's instructions. Total RNA ( $1 \mu \mathrm{g})$ from each sample was resuspended in $20 \mu \mathrm{l}$ of the reaction buffer. Commercially available PCR primers for TIMP-1, MMP-2 and glyceraldehyde-3-phosphate dehydrogenase (GAPDH) were purchased from Purigo Biotech, Inc. (Taipei, Taiwan, R.O.C.) and contained the following sequences: TIMP-1, sense 5'-CATGGAAAGCCTCTGTGGA TATG-3', antisense 5'-GATGTGCAAATTTCCGTTCCTT-3'; MMP-2, sense 5'-GATACCCTCAAGAACATGCAGAAG TT-3', antisense 5'-ACATCTTGGCTTCCGCATG-3'; and GAPDH, sense 5'-CCCTTCATTGACCTCAACTACATGG3', antisense 5'-CATGGTGGTGAAGACGCCAG-3'. The amplified PCR products were electrophoresed at $75 \mathrm{~V}$ through $2 \%$ agarose gel for $1 \mathrm{~h}$. The PCR products were visualized by ethidium bromide staining, and the relative amount of messenger RNA transcripts was scanned by the Imaging Master. Densitometric analysis of the captured image was normalized for GAPDH content.
Two-dimensional polyacrylamide gel electrophoresis (2-DE). Liver tissue $(50 \mathrm{mg}$ ) was ground with Medimechine (BD Biosciences, Franklin Lakes, NJ); $10 \mathrm{mg}$ was dissolved in $200 \mu 1$ of lysis buffer $\{7 \mathrm{M}$ urea/4 M thiourea/4\% (3-[(3cholamidopropyl) dimethylammonio]-1-propanesulfonate)\} (CHAPS) and sonicated for 10 cycles of $1 \mathrm{sec}$. The sample was kept shaking for $30 \mathrm{~min}$ at $25^{\circ} \mathrm{C}$, and centrifuged for $15 \mathrm{~min}$ at $13000 \mathrm{x}$ g. Protein $(500 \mu \mathrm{g})$ and rehydration buffer [containing $7 \mathrm{M}$ urea, 4\% CHAPS, $2 \mathrm{M}$ thiourea, $0.5 \%$ immobilized $\mathrm{pH}$ gradient (IPG) buffer, and $65 \mathrm{mM}$ dithiothreitol (DTT)] was rehydrated onto $18 \mathrm{~cm}, \mathrm{pH}$ 4.0-7.0 (linear) IPG strips using IPGphor II (GE Healthcare, Uppsala, Sweden) at $30 \mathrm{~V}$ for $12 \mathrm{~h}$. After the completion of isoelectric focusing (first dimensional separation), the proteins on the IPG strips were then incubated for $15 \mathrm{~min}$ in an equilibration buffer containing $6 \mathrm{M}$ urea, $2 \%$ SDS, $30 \%$ glycerol, $13 \mathrm{mM}$ DTT, and $50 \mathrm{mM}$ Tris ( $\mathrm{pH}$ 8.8). The IPG strips were further equilibrated in a similar equilibration buffer, in which DTT was replaced with $2.5 \%$ iodoacetamide. The second dimensional separation was performed using a $12.5 \%$ acrylamide gel and run at $10 \mathrm{~mA} /$ gel for $20 \mathrm{~min}$ followed by $100 \mathrm{~mA} / \mathrm{gel}$ until the dye front reached the bottom of the gel. After electrophoresis, the gel was fixed in fix solution (10\% methanol and 7\% acetic acid) and stained with SYPRO Ruby ${ }^{\mathrm{TM}}$ (Perkin-Elmer, Boston, MA, USA) solution. The gel image was scanned with the Typhoon $9200^{\mathrm{TM}}$ (Amersham Pharmacia, Piscataway, NJ) and analyzed with PDQuest ${ }^{\mathrm{TM}}$ (Bio-Rad).

The protein spots detected on the $2 \mathrm{D}$ gel were manually excised from the gel. The gel spots were rehydrated with $50 \mathrm{mM}$ dithioerythritol in $25 \mathrm{mM}$ ammonium bicarbonate, $\mathrm{pH} 8.5$, at $37^{\circ} \mathrm{C}$ for $1 \mathrm{~h}$, and subsequently alkylated with $100 \mathrm{mM}$ iodoacetamide in $25 \mathrm{mM}$ ammonium bicarbonate, $\mathrm{pH} 8.5$, at room temperature for $1 \mathrm{~h}$. The spots were then washed twice with $50 \%$ acetonitrile in $25 \mathrm{mM}$ ammonium bicarbonate, $\mathrm{pH} 8.5$, for $15 \mathrm{~min}$ each time, dehydrated with acetonitrile for $5 \mathrm{~min}$, dried, and rehydrated with a total of $100 \mathrm{ng}$ of sequencing grade, modified trypsin (Promega,

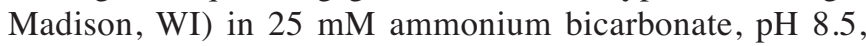
at $37^{\circ} \mathrm{C}$ for $16 \mathrm{~h}$. Following digestion, tryptic peptides were extracted twice with $50 \%$ acetonitrile containing $5 \%$ formic acid for 15 min each time with moderate sonication. The extracted solutions were pooled and evaporated to dryness under vacuum.

MALDI MS and MS/MS analysis - samples were premixed $1: 1$ with matrix solution $(5 \mathrm{mg} / \mathrm{ml} \alpha$-cyano-4-hydroxycinnamic acid in $50 \%$ acetonitrile, $0.1 \% \mathrm{v} / \mathrm{v}$ trifluoroacetic acid and $2 \% \mathrm{w} / \mathrm{v}$ ammonium citrate) and spotted onto the 96-well format MALDI sample stage. Data directed acquisition on the Q-TOF Ultima ${ }^{\mathrm{TM}}$ MALDI instrument was fully automated with a predefined probe motion pattern and peak intensity threshold for switching over from MS survey scan to MS/MS, and from one MS/MS to another. Within each well, parent ions meeting the predefined criteria (any peak within the $m / z 800-3000$ range with intensity above 10 count \pm include/exclude list) were selected for collision-induceddissociation MS/MS using argon as the collision gas and a mass dependent $\pm 5 \mathrm{~V}$ rolling collision energy until end of probe pattern was reached, starting from the most intense peak. The instrument was externally calibrated to less than $5 \mathrm{ppm}$ accuracy over the mass range of $\mathrm{m} / \mathrm{z}$ 800-3000 using 

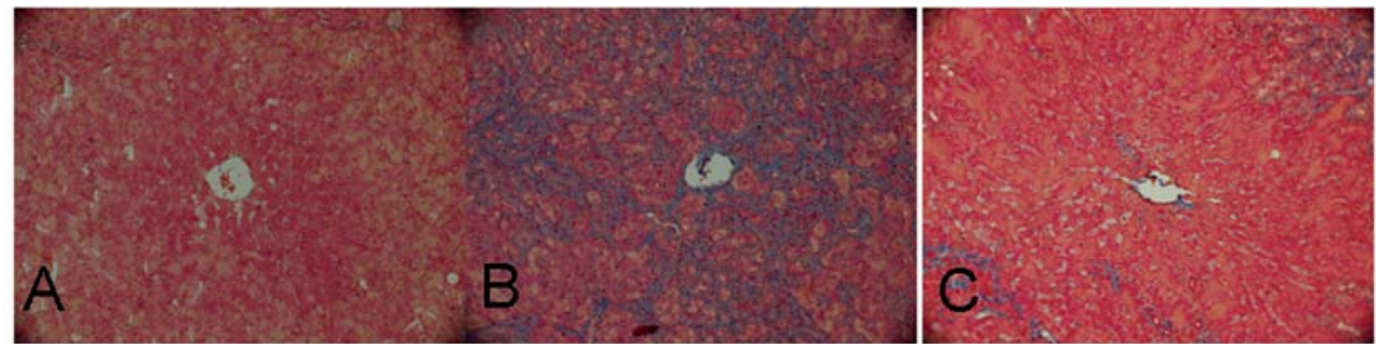

Figure 1. Light microscopic analysis of rat liver sections of sham rats and rats after bile duct ligation treatment with or without YCHT administration. Sham group rat liver (A); BDL surgery alone (B) and BDL concomitant treatment with YCHT $(250 \mathrm{mg} / \mathrm{kg})(\mathrm{C})$ for 27 days. Paraffin-embedded sections were stained with Masson's trichrome. Original magnification x200.

A
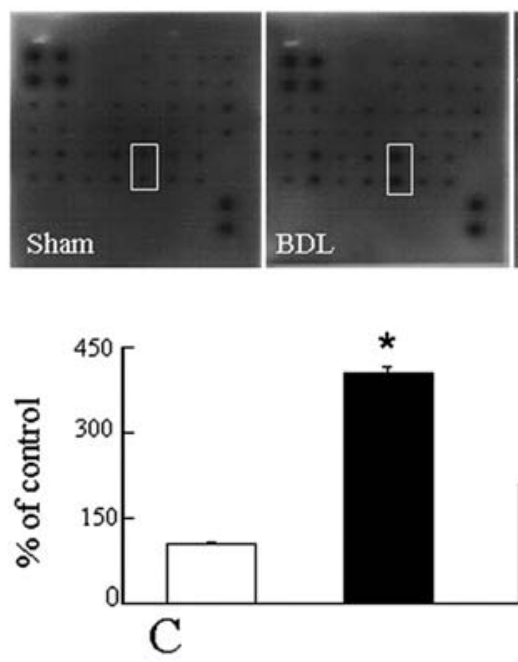

Pos

Pos

GM-CSF

GM-CSF

Leptin

Ieptin

Blank

Blank

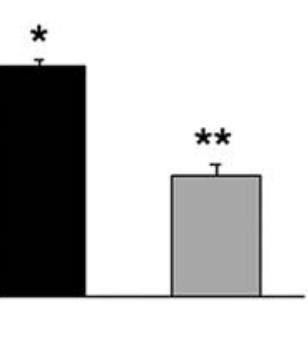

Pos Neg Neg

Pos Neg Neg

IFN- $\gamma \quad \mathbb{L}-1 \alpha \quad$ I $1 \beta$

IFN- $\gamma$ 표 $\alpha$ 표 $1 \beta$

MCP-1 MIP-3 $\alpha \quad \beta-N G F$

MCP-1 MIP-3 $\alpha \quad \beta-$ NGF

Blank.

Blank
Blanik.

Blank
Blank

Blank
$\mathrm{B}$
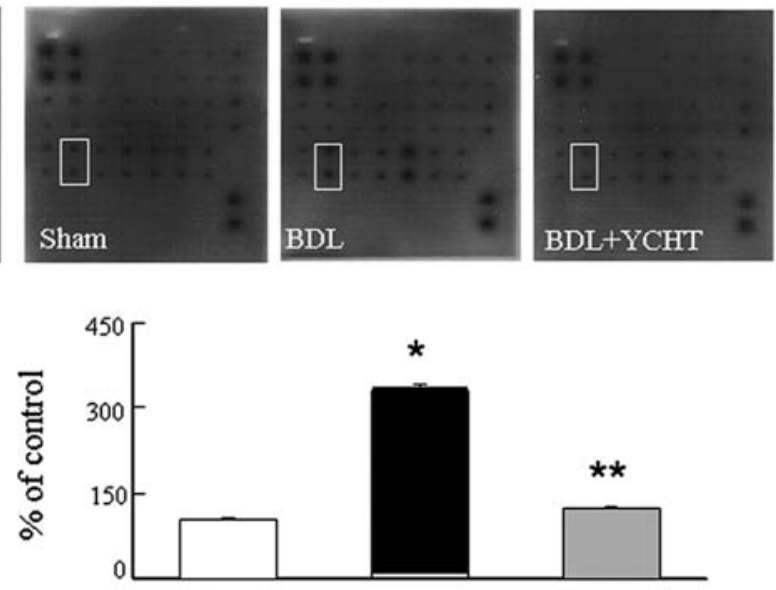

$\begin{array}{llll}\text { CINC-2 } & \text { CINC-3 } & \text { CNTF } & \text { Fractalkine } \\ \text { CINC-2 } & \text { CINC-3 } & \text { CNTF } & \text { Fractalkine } \\ \text { II-4 } & \text { IL-6 } & \text { IL-10 } & \text { LIX } \\ \text { II-4 } & \text { IL-6 } & \text { I-10 } & \text { LIX } \\ \text { TMMP-1 } & \text { TNF- } \alpha & \text { VEGF } & \text { Blank } \\ \text { TIMP-1 } & \text { TNF- } \alpha & \text { VEGF } & \text { Blank } \\ \text { Blank } & \text { Blank } & \text { Blank } & \text { Pos } \\ \text { Blank } & \text { Blank } & \text { Blank } & \text { Pos }\end{array}$

Figure 2. Detection of cytokine expression from serum of rats induced by BDL and YCHT treatment groups. Cytokine array patterns were scanned, and the intensities of signals were quantitated by densitometry. Data are presented as mean $\pm \mathrm{SEM} .{ }^{*} \mathrm{P}<0.05$ compared with the sham group; ${ }^{* *} \mathrm{P}<0.05$ compared with the BDL group. (C) rat cytokine array layout.

sodium iodide and polyethylene glycol 200,600, 1000 and 2000 mixtures, and further adjusted with Glu-Fibrinopeptide B as the near-point lock mass calibrant during data processing. At a laser firing rate of $10 \mathrm{~Hz}$, individual spectra from 5 second integration periods acquired for each of the MS survey and MS/MS performed were combined, smoothed, deisotoped (fast option) and centroided using the Micromass Protein Lynx $^{\mathrm{TM}}$ Global Server (PGS) 2.0 data processing software (Cary, NC). The combined peptide mass fingerprinting (PMF) and MS/MS ion meta data were searched in concert against the specified protein database within the PGS 2.0 workflow. Alternatively or additionally, the PMF and individual MS/MS ion data were output as Mascot-searchable .txt file and .pkl files for independent searches against Swiss-Prot database using the Mascot program.
Statistics. Results are expressed as means \pm SEM. Statistical analysis were performed using one-way analysis of variance (ANOVA) followed by Student-Newman-Keuls' multiple range test. Significance was determined at $\mathrm{P}<0.05$.

\section{Results}

YCHT improved liver histopathology and serum markers of $B D L$ rats. Histological examination showed a significant change in the profile of collagen fiber deposition in the liver sections of BDL rats compared with rats that received YCHT treatment (Fig. 1). Liver sections from the sham rats showed no fibrosis (Fig. 1A). After BDL surgery, liver of rats produced typical histological changes characterized by architecture disruption and fibrosis formation (Fig. 1B). The BDL rats that 

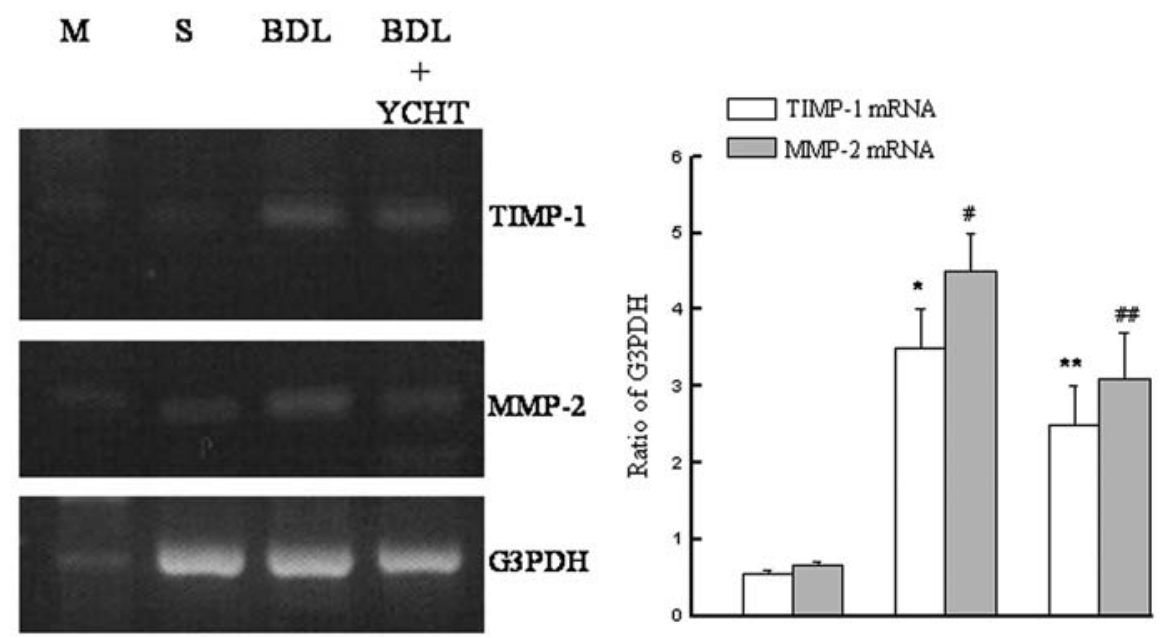

Figure 3. Amplification of hepatic TIMP-1 and MMP-2 mRNA by RT-PCR from livers of rats: sham, in rats after BDL alone, with BDL and YCHT (250 mg/kg) administration after surgery for 27 days. Using cDNA equivalents of $10 \mathrm{ng}$ RNA, samples were amplified for 32 cycles using specific TIMP-1 primers; 30 cycles using specific MMP-2 primers. All values were corrected for differences in loading, and are expressed in arbitrary units and refer to mean \pm SEM. ${ }^{*}, \# \mathrm{P}<0.05$ compared with the sham group; ${ }^{* *}, \#$ P $<0.05$ compared with the BDL group.

were treated with YCHT (250 mg/kg) (Fig. 1C), had significantly less histological collagen accumulation. Obviously, the cytokine expression down-regulated in respect to YCHT treated and displayed a distinct pattern in which BDL-induced TIMP-1 (Fig. 2A) and MCP-1 (Fig. 2B) were significantly upregulation.

YCHT reduced levels of TIMP-1 and MMP-2 transcript in liver of BDL rats. We analyzed expression of TIMP-1 and MMP-2 genes related to extracellular matrix formation. As shown in Fig. 3, TIMP-1 mRNA expression was induced 4 -fold after bile duct ligation. Changes in expression of TIMP-1 were accompanied by a detectable increase in MMP-2 mRNA. BDL rats concomitant with YCHT treatment, caused a marked reduction in the level of expression of the genes transcript. However, these experiments did not reveal which liver cell type is responsible for the induction mRNAs expression.

Proteomics. Using 2-DE, the differential expression of molecules retrieved from liver tissues and serum of experimental animals were analyzed, respectively. Eleven protein spots in liver tissue and six protein spots of the animals were analyzed (Fig. 4). These 17 protein spots were retrieved from the 2-DE gel and processed using in gel enzymatic digestion and MALDI-TOF, which gave rise to the final identification (Tables I and II). From the 11 identified proteins in the liver, 5 showed a significant reduction and 6 were significantly upregulated in BDL rats. From the 6 identified proteins in the serum fraction, 4 showed a significant reduction and 2 were significantly up-regulated in BDL rats. Among the variation proteins, enzymes related to the lipid metabolism (glycoprotein 330, apolipoprotein A-I precursor) and cytoskeleton (keratin 8 and 19) were affected. Additionally, cytoplasmic dynein heavy chain 1 (DYHC-1) and senescence marker protein (SMP-30). It should be pointed out these changes in proteins level were shown as a significance statistic or reached the usual threshold of 2-fold up- or down-regulation in YCHT treatment groups.

\section{Discussion}

YCHT has been used in the treatment of various liver disease models (7-9). Here, we have applied proteomics to enhance our understanding of the fundamental aspects of how YCHT exerts its hepatoprotection effect on bile duct ligated rats to provide potential insights which may impact medical practice. To our knowledge, this is the first description of liver proteome alterations after BDL surgical induction and YCHT supplement. In the following section, the possible function of these proteins, and particularly those that showed changes after YCHT administration, will be discussed.

We initially analyzed the expression profiles of serum proteins in the BDL model after YCHT administration using 2-DE. MS revealed that serum levels of ApoA-I, haptoglobin, plectin-1 and glycoprotein 330 were decreased in the BDL group compared with sham group (Fig. 4C and Table II). Several studies have shown that the ApoA-I level correlates with changes in hepatocellular function, and a low level of ApoA-I indicates severe liver cell injury, which has been confirmed in recent proteomic studies of hepatitis B virus infected liver and hepatocellular carcinoma $(14,15)$. Upregulation of ApoA-I in BDL rats after YCHT administration indicated enhanced triglyceride metabolism, which may link ApoA-I deficiency to the pathophysiology of liver fibrosis with bile duct ligation.

Haptoglobin is a $\alpha 2$-glycoprotein synthesized by the liver which is down-regulated during the course of BDL. In this study, the fact that YCHT increased rather than decreased haptoglobin levels may reflect the antioxidant properties of YCHT (10). In fact, haptoglobin may function as an antioxidant and act as a potent immunosuppressor of lymphocyte function which modulates helper-T cell type 1 and 2 balance within the body (16).

Plectin is a major intermediate filament (IF)-based cytolinker protein that stabilizes cells and tissues mechanically, regulates actin filament dynamics, and serves as a signaling molecule. It has been reported that loss of plectin is a cause of muscular dystrophy with epidermolysis bullosa (17). As 
A.

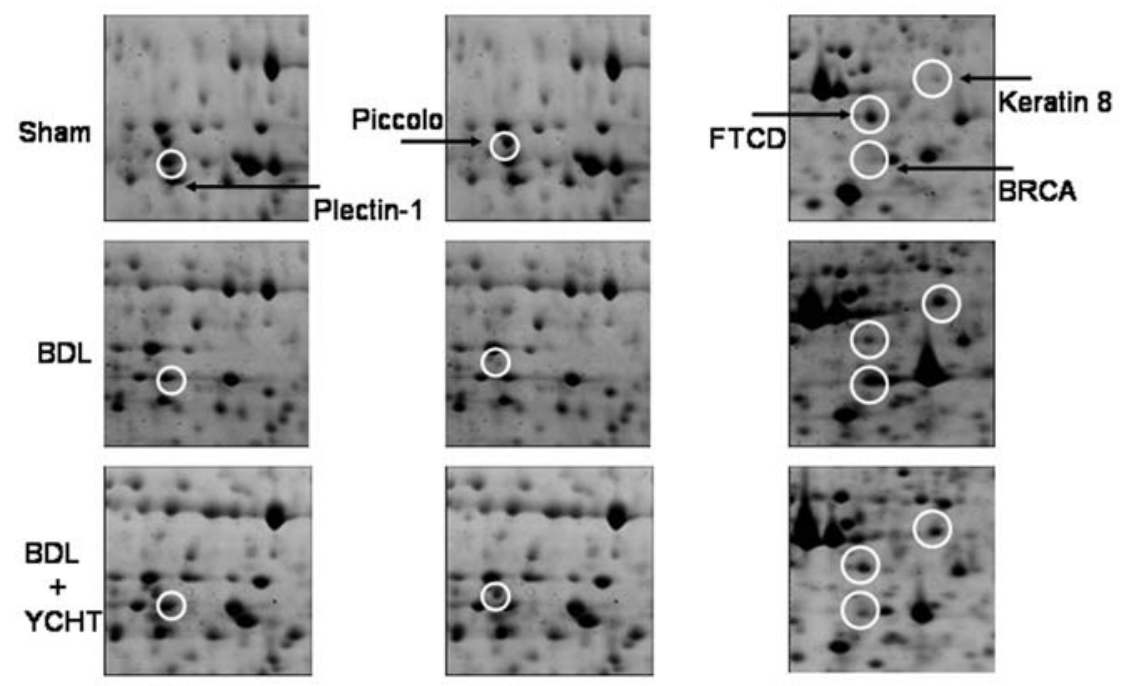

B.
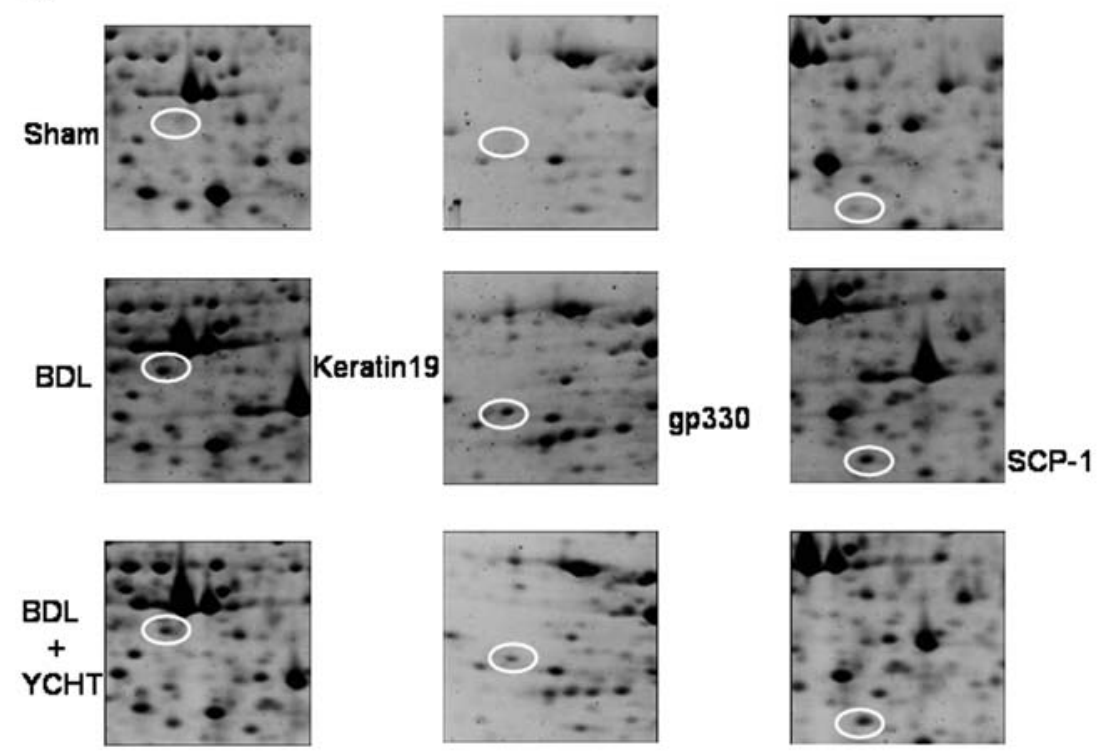

c.
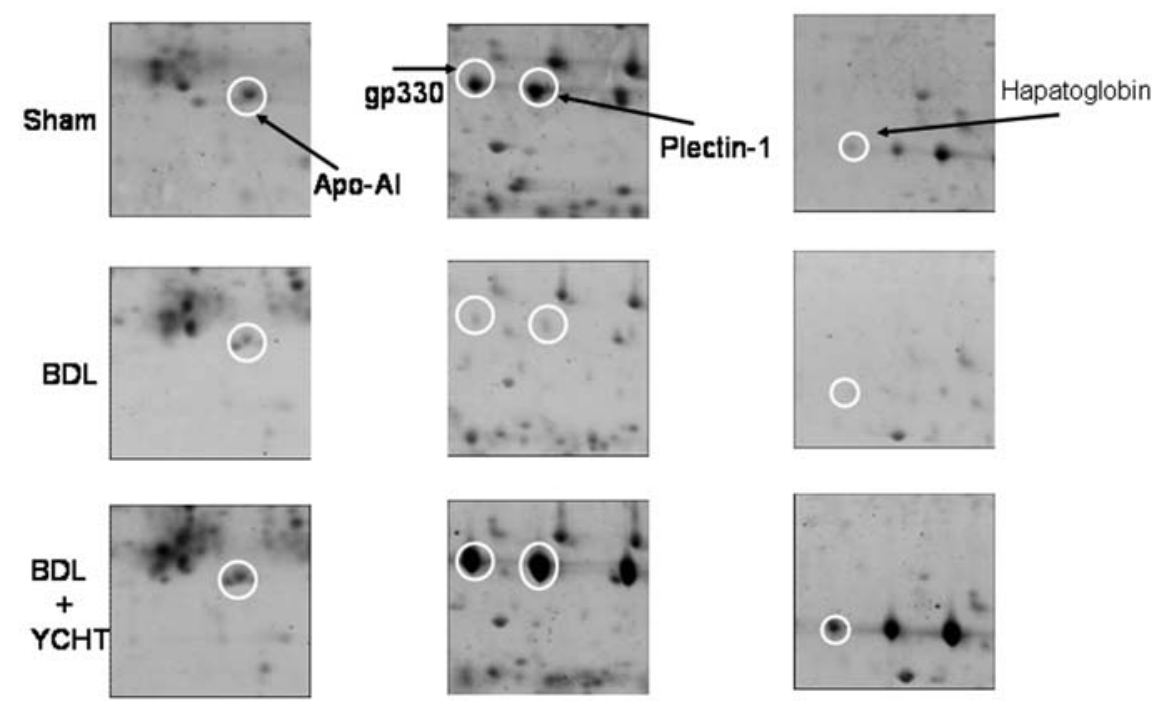

Figure 4. Partial 2D images of liver proteins from sham, BDL and animals receiving YCHT treatment. The protein samples were analyzed as stated under the Materials and methods. Examples of spots representing proteins whose level changed due to the YCHT treatment are highlighted, and the measured protein levels for plectin-1, piccolo, keratin 8, formiminotransferase-cyclodeaminase (FTCD) and breast cancer type 2 susceptibility protein homolog (BRCA) are shown in (A); keratin 19, low-density lipoprotein receptor-related protein 2 precursor (gp330) and synaptonemal complex protein 1 (SCP-1) are shown in (B); partial 2D images of serum proteins from sham, BDL and animals receiving YCHT treatment. Plectin-1, haptoglobin precursor, low-density lipoprotein receptor-related protein 2 precursor (gp330) and ApoA-I are shown in (C). 


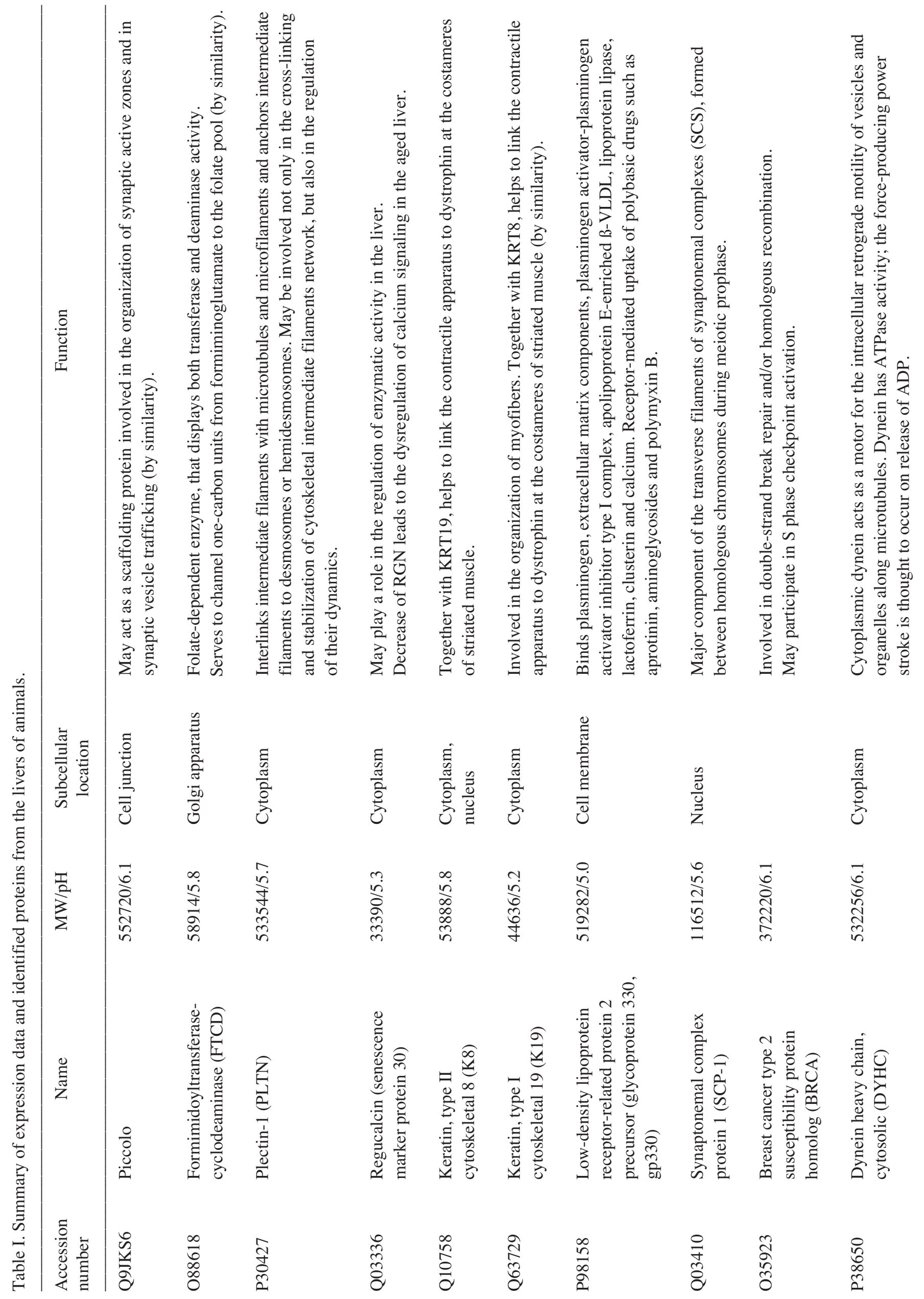




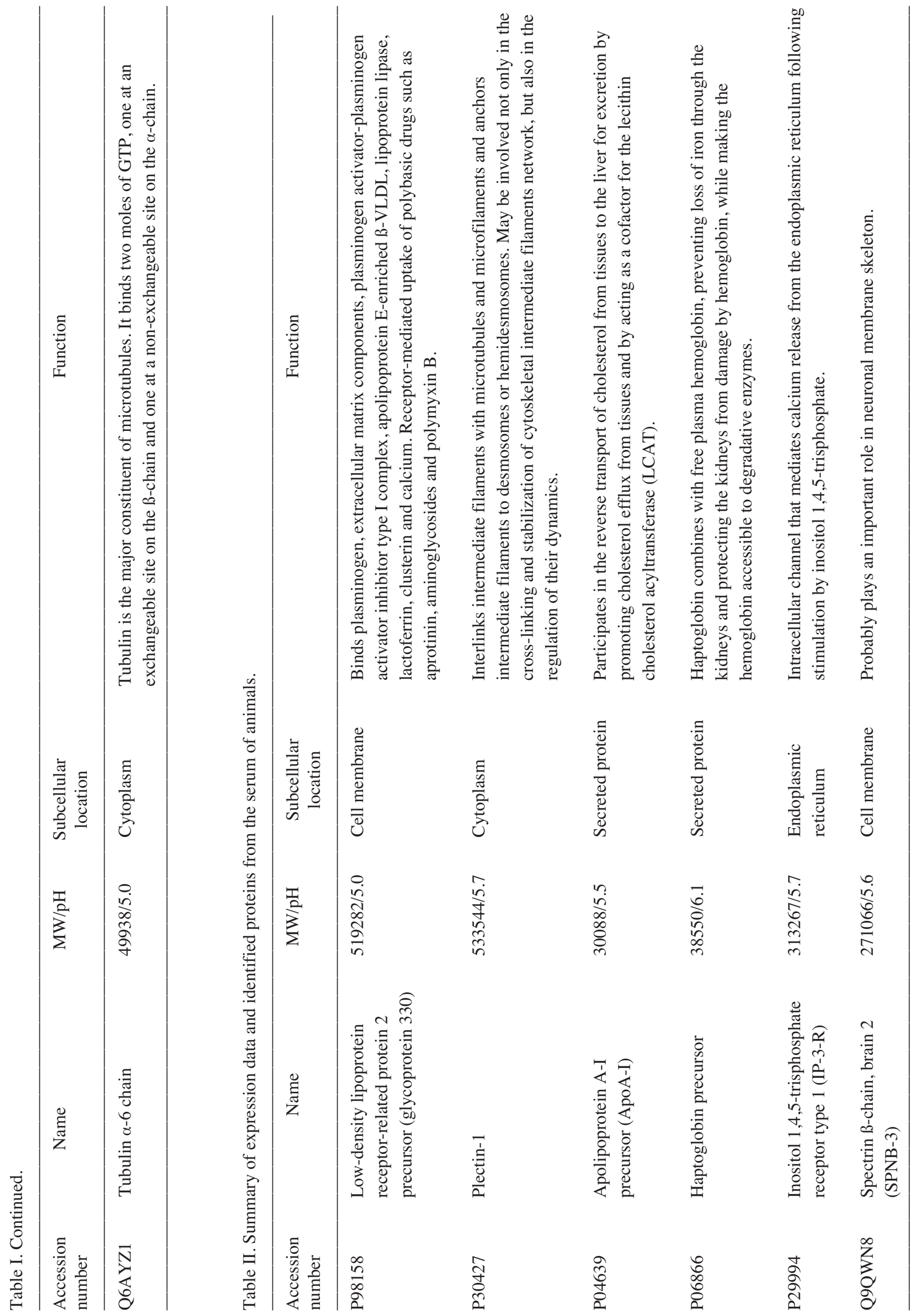


shown in Fig. 4A and C, plectin-1 expression was significantly increased in both serum and liver in BDL rats after YCHT administration. One possible explanation was that because of decreased plectin-1 expression in the liver, the rigidity of IF networks was reduced and filaments were more loosely bound, or not bound at all, to the outer membrane in BDL rats compared with the sham group. YCHT may disrupt this event by enhancing soluble plectin to act as a cross-linker of IF in the cytoplasm, but further studies are required to elucidate detailed mechanisms.

Bile duct epithelia revealed a more complex keratin expression pattern than tumor cells since they expressed keratins K7 and K19 in addition K8 and K18 (18). In addition, BDL-induced biliary atresia characterized by intense leukocyte infiltration of liver combined with retention of toxic bile acid as hepatocyte damage and mild to severe fibrosis (19). In cholestatic liver disease, keratins not only act as skeletal proteins providing mechanical stability, but are also involved in the execution of apoptosis since hyperphosphorylated keratins activate caspases (20). There was a striking overexpression of keratin in the bile duct epithelia in response to BDL, and in hepatocytes in the proximity of bile infarcts (21). Our study demonstrated that YCHT decreased keratin 8 and 19 protein levels (Fig. 4B) associated with better tolerance to toxic stress, and therefore was not deleterious to hepatocytes. YCHT is considered as a protective agent of defense responses to hepatic apoptosis after treatment in BDL rats (13).

In this study, serum MCP-1 levels were significantly increased in BDL rats with hepatic fibrosis compared with YCHT treatment and sham groups (Fig. 2). There was also a significant correlation between serum MCP-1 and TIMP-1 levels in BDL rats. The high serum levels of MCP-1 may contribute to the promotion of inflammation and fibrosis of $\mathrm{BDL}$ rats. YCHT treatment prevented $\mathrm{MCP}-1$ secretion. Moreover, inhibiting its effects might present an opportunity to alter the progression of liver dysfunction in BDL rats with fibrosis. The net deposition of interstitial collagens in chronic hepatic fibrosis occurs not only as a consequence of increased fibrillar collagen synthesis, but also as a result of decreased degradation by collagenases. Although our RT-PCR analysis does not provide information on the relative activity of the MMP and TIMP proteins, it does indicate how these may be affected by measuring the expression of the corresponding mRNA. Treatment with YCHT was effective in reducing the level of TIMP-1 and MMP-2 in BDL rats (Fig. 3), and resulted in a greater improvement in the histological analysis (Fig. 1).

In conclusion, this study demonstrated that YCHT exerts dramatic antifibrotic action in BDL-induced hepatic fibrosis, and this effect may be caused at least in part by its inhibitory action on ECM gene expression. On the other hand, proteome analysis revealed quantitative changes in rat hepatic proteins following treatment with YCHT. This herbal remedy triggered alterations in lipid metabolism, and is associated with changes in cellular stress proteins involved in cytoskeletal filament and apoptosis response.

\section{Acknowledgements}

This work was supported by grant no. CCMP95-RD-207 from the Committee on Chinese Medicine and Pharmacy, Department of Health, and NSC95-2320-B-182-052 from the
National Science Council, Taiwan, R.O.C. and CMRPD150181 from the Chang Gung Memorial Hospital, Taoyuan, Taiwan, R.O.C.

\section{References}

1. Friedman SL: Liver fibrosis - from bench to bedside. J Hepatol 38: S38-S53, 2003.

2. Arthur MJ: Fibrogenesis II. Metalloproteinases and their inhibitors in liver fibrosis. Am J Physiol 279: G245-G249, 2000.

3. Anderson NL, Esquer-Blasco R, Richardson F, Foxworthy P and Eacho P: The effects of peroxisome proliferators on protein abundances in mouse liver. Toxicol Appl Pharmacol 137: 75-89, 1996.

4. Arce A, Aicher L, Wahl D, et al: Changes in the liver protein pattern of female Wistar rats treated with the hypoglycemic agent SDZ PGU 693. Life Sci 63: 2243-2250, 1998.

5. Fountoulakis M, Berndt P, Boelsterli UA, Crameri F, Winter M, Albertini S and Suter L: Two-dimensional database of mouse liver proteins: changes in hepatic protein levels following treatment with acetaminophen or its nontoxic regioisomer 3acetamidophenol. Electrophoresis 21: 2148-2161, 2000.

6. Fountoulakis M, De Vera MC, Crameri F, Boess F, Gasser R, Albertini S and Suter L: Modulation of gene and protein expression by carbon tetrachloride in the rat liver. Toxicol Appl Pharmacol 183: 71-80, 2002.

7. Sakaida I, Tsuchiya M, Kawaguchi K, Kimura T, Terai S and Okita K: Herbal medicine Inchin-ko-to (TJ-135) prevents liver fibrosis and enzyme-altered lesions in rat liver cirrhosis induced by a choline-deficient L-amino acid-defined diet. J Hepatol 38: 762-769, 2003

8. Inao M, Mochida S, Matsui A, et al: Japanese herbal medicine Inchin-ko-to as a therapeutic drug for liver fibrosis. J Hepatol 41: 584-591, 2004.

9. Imanishi Y, Maeda N, Otogawa K, Seki S, Matsui H, Kawada N and Arakawa T: Herb medicine Inchin-ko-to (TJ-135) regulates PDGF-BB-dependent signaling pathways of hepatic stellate cells in primary culture and attenuates development of liver fibrosis induced by thioacetamide administration in rats. J Hepatol 41: 242-250, 2004.

10. Lee TY, Chang HH, Chen JH, Hsueh ML and Kuo JJ: Herb medicine Yin-Chen-Hao-Tang ameliorates hepatic fibrosis in bile duct ligation rats. J Ethnopharmacol 109: 318-324, 2007.

11. Yamamoto M, Ogawa K, Morita M, Fukuda K and Komatsu Y: The herbal medicine Inchin-ko-to inhibits liver cell apoptosis induced by transforming growth factor beta 1. Hepatology 23: 552-559, 1996.

12. Yamamoto M, Miura N, Ohtake N, et al: Genipin, a metabolite derived from the herbal medicine Inchin-ko-to, and suppression of Fas-induced lethal liver apoptosis in mice. Gastroenterology 118: 380-389, 2000.

13. Lee TY, Chang HH, Wu MY and Lin HC: Yin-Chen-Hao-Tang ameliorates obstruction-induced hepatic apoptosis in rats. J Pharm Pharmacol 59: 583-590, 2007.

14. He QY, Lau GK, Zhou Y, Yuen ST, Lin MC, Kung HF and Chiu JF: Serum biomarkers of hepatitis B virus infected liver inflammation: a proteomic study. Proteomics 3: 666-674, 2003.

15. Steel LF, Shumpert D, Trotter M, et al: A strategy for the comparative analysis of serum proteomes for the discovery of biomarkers for hepatocellular carcinoma. Proteomics 3: 601-609, 2003.

16. Sadrzadeh SM and Bozorgmehr J: Haptoglobin phenotypes in health and disorders. Am J Clin Pathol 121: S97-S104, 2004.

17. McLean WH, Pulkkinen L, Smith FJ, et al: Loss of plectin causes epidermolysis bullosa with muscular dystrophy: cDNA cloning and genomic organization. Genes Dev 10: 1724-1735, 1996.

18. Moll R, Franke WW, Schiller DL, Geiger B and Krepler R: The catalog of human cytokeratins: patterns of expression in normal epithelia, tumors and cultured cells. Cell 31: 11-24, 1982.

19. Kobayashi H, Narumi S, Tamatani T, Lane GJ and Miyano T: Serum IFN-inducible protein-10: a new clinical prognostic predictor of hepatocyte death in biliary atresia. J Pediatr Surg 34: 308-311, 1999.

20. MacFarlane M, Merrison W, Dinsdale D and Cohen GM: Active caspases and cleaved cytokeratins are sequestered into cytoplasmic inclusions in TRAIL-induced apoptosis. J Cell Biol 148: 1239-1254, 2000

21. Fickert P, Trauner M, Fuchsbichler A, Stumptner C, Zatloukal K and Denk H: Cytokeratins as targets for bile acid-induced toxicity. Am J Physiol 160: G491-G499, 2002. 